\title{
Evaluation of the Grenada Sports for Health Program
}

\author{
Satesh Bidaisee*1and Calum Macpherson ${ }^{2}$ \\ ${ }^{1}$ Department of Public Health and Preventive Medicine, St. George's University, Windward Islands Research and Education, Grenada \\ ${ }^{2}$ Department of Microbiology, St. George's University, Windward Islands Research and Education Foundation, Grenada
}

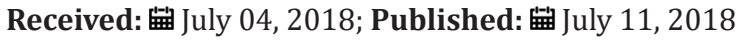

*Corresponding author: Satesh Bidaisee, Department of Public Health and Preventive Medicine, School of Medicine, St. George's University, Windward Islands Research and Education,Grenada,West indies

\begin{abstract}
Objective: The study served to measure basic health outcome measures to help guide the continued implementation of the community exercise component of the Grenada Sports for Health program.

Design \& Methods: The study population consisted of Grenadian citizens enrolled in three different community exercise programs as part of the Royal Grenada Police Force, Point Saline and La Sagesse, Grenville, Gouyave and Tanteen community exercise program. Initial data collection for this prospective cohort study began during March of 2011 and continued data collection through quarterly assessments was continued to June/ July 2014 and June/July 2016. The health indicators for the Sports for Health program were designed to monitor and analyse program participants' physical health indicators, such as Body Mass Index (BMI), Waist to Hip ratio over time to determine if their participation in the community training program was promoting health benefits by reducing risk factors for non-communicable chronic diseases.

Results: During the baseline evaluation period in March, 2011, complete data sets were obtained for 427 participants. During the evaluation period of March 2014, 337 complete data sets were collected from participants from 2011 and during June/July 2016 evaluation, 264 complete data sets were obtained. The BMI, Waist, hip, and waist: hip ratio is presented in Table 1. BMI and Waist: Hip ratio using a Student's T-test ( $\alpha=0.05$ ) demonstrated a significant difference between 2011 and 2016 measures (Table 1).
\end{abstract}

Conclusion: Participants have demonstrated a significant and positive difference in physical health indicators over three years of participation in the Sports for Health program.

\section{Introduction}

Over the past few decades, a rise in non-communicable diseases globally and especially in the developing nations has occurred. This may be due to socioeconomic disparities[1] and more so the increasing income inequality [2], as well as the westernization of diets, combined with a decrease in physical activity. Based on WHO report, non-communicable diseases (NCD) are responsible for $63 \%$ of deaths globally, with nearly $80 \%$ of NCD deaths occurring in low and middle- income nations[3].The greatest risk factors for NCD are tobacco use, physical inactivity, alcohol abuse and unhealthy diet. Physical activity is a critical component for decreasing overall mortality and incidence of NCD[3-5].Furthermore, exerciseimproves cognition in older adults[6-9].Studies show communitybased programs to be effective in managing and improving the outcomes of non-communicable diseases, especially cardiovascular disease, hypertension and obesity[10,11]. Community based programs also show an increase in participation and a decrease in health care costs[12].

The use of community based approaches to health improvement is not new and serves as a mutli-pronged approach to create a positive change. A community approach not only engages in healthy behaviours, but also empowers members of the community to make lasting and effective changes[13].The community exercise program in Grenada provides community based exercise opportunities to help reduce the burden of NCDs. The program is run by the Royal Grenadian Police Force at fivesites around the island of Grenada. The program is focused on engaging adults in a more active lifestyle by delivering different exercise activities to a group five days a week. This study aimed to evaluate the efficacy of the sports for health program for improving health outcomes using a community based intervention.

\section{Design and Methods}

The study population consisted of Grenadian citizens enrolled in five different community exercise programs as part of the Royal Grenada Police Force, Fitness Club in Point Saline and La Sagesse, Grenville, Gouyave and Tanteen. The community exercise programs offered aerobic exercises, ranging from dancing, fast track, jogging, running, and strength training.Initial data collection for this prospective cohort study began during March of 2011 and continued data collection through quarterly assessments was carriedthrough June/July 2016. The Sports for Health program was designed to monitor and analyse program participants' physical health indicators, such as Body Mass Index (BMI), Waist to Hip ratio over 
time, to determine if their participation in the community training program was improving health indicators. The measurements for hip and waist were collected using a tape measure. The weight was collected using a standard mechanical scale. Data were recorded on survey forms at the fitness centres.

\section{Results}

During the baseline evaluation period in March, 2011, complete data sets were obtained for 427 participants. During the evaluation period of March 2014, 337 complete data sets were collected from participant's from 2011 and 264 complete data sets in 2016. The BMI, Waist, hip, and waist: hip ratio is presented in Table 1. BMI and Waist: Hip ratio using a Student's T-test $(\alpha=0.05)$ demonstrated a significant difference for both measures between 2011 and 2014 . There was no demonstrated difference between 2014 and 2016. The number of participants available for the study in 2011, 2014 and 2016 decreased over time. In 2014 there was a loss of 90 participants and a further loss of 73 participants in 2016, resulting in an overall loss of $40 \%$ of initial participants.Overall changes in participant measures of waist, hip and weight measurements reported a decrease in all measurements in 2014 with maintenance in 2016. The change in BMI measurement between the group in 2011 and 2014 showed a decrease of 4.9, then maintenance in 2016. The waist to hip ratio reduction of 0.04 was observed between 2011 and 2014 and also maintained in 2016 see Figure 1.The comparison measures of BMI and waist to hip ratio between 2011 and 2014 were significant and had a p- value $<0.05$, with no observable difference between 2014 and 2016.

Table 1: Comparing physical health indicators between 2011, 2014 and 2016.

\begin{tabular}{|c|c|c|c|}
\hline \multicolumn{4}{|c|}{ Measured physical health indicators between 2011, 2014 and 2016} \\
\hline & $\mathbf{2 0 1 1}$ & $\mathbf{2 0 1 4}$ & $\mathbf{2 0 1 6}$ \\
\hline Number of Participants & 427 & 337 & 264 \\
\hline Height (cm) & $164 \pm 9$ & $164 \pm 7.6$ & $165.6 \pm 10.3$ \\
\hline Weight (kg) & $80.9 \pm 14.9$ & $68.4 \pm 19$ & $68.2 \pm 16.9$ \\
\hline BMI & $30.3 \pm 7.1$ & $25.4 \pm 6.8$ & $25.2 \pm 6.1$ \\
\hline Waist (cm) & $91.2 \pm 12.6$ & $82.5 \pm 19$ & $83.7 \pm 12.5$ \\
\hline Hip (cm) & $106.4 \pm 11.8$ & $99.3 \pm 15.7$ & $99.5 \pm 10.7$ \\
\hline Waist: Hip Ratio (WHR) & $0.86 \pm 0.07$ & $0.82 \pm 0.08$ & $0.82 \pm 0.05$ \\
\hline \multicolumn{4}{|c|}{ All data, except WHR, reported as mean \pm standard deviation. } \\
\hline \multicolumn{4}{|c|}{ P<0.05 for comparisons of BMI and Waist: Hip ratio } \\
\hline
\end{tabular}

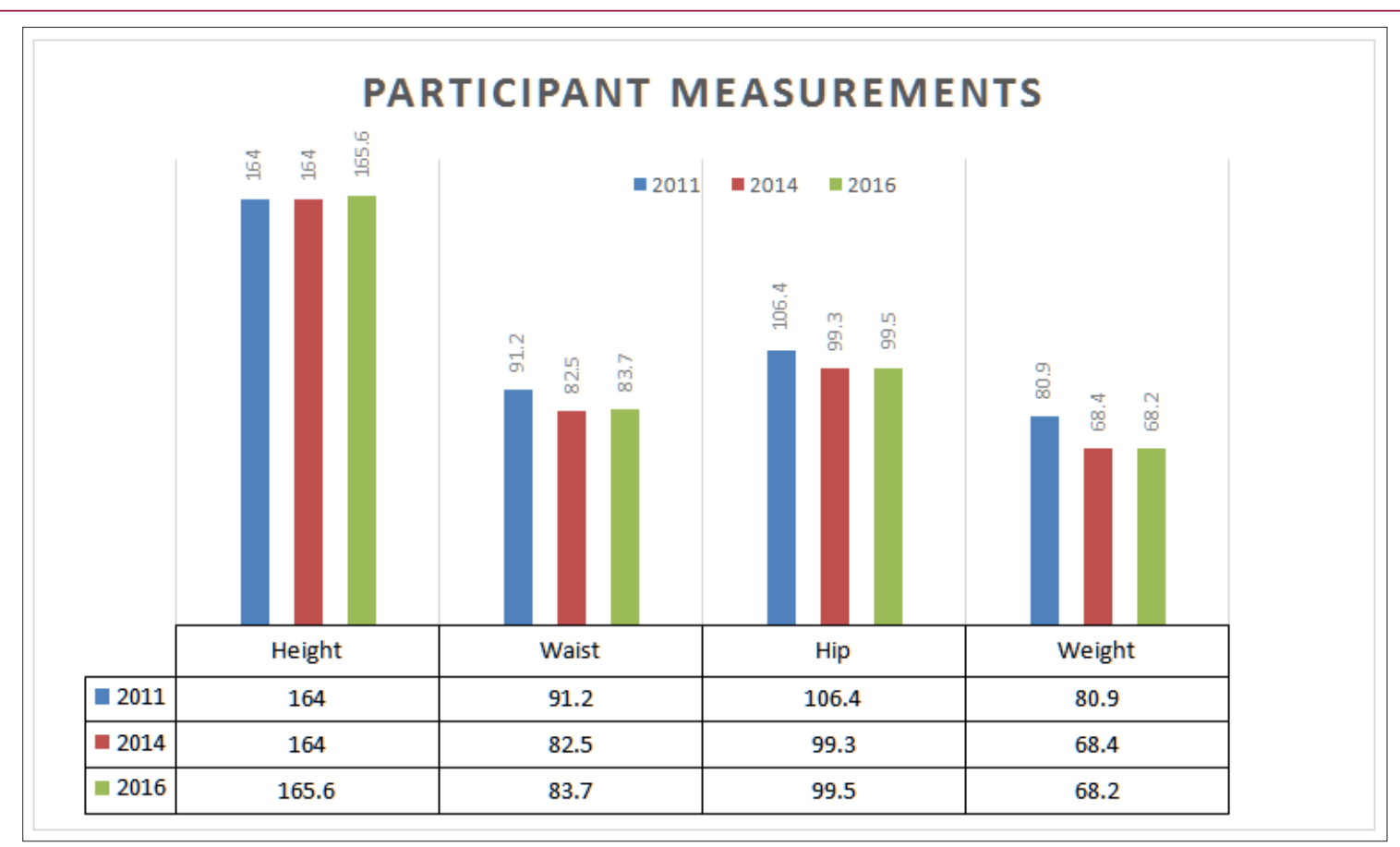

Figure 1: Participant Measurements.

\section{Discussion}

The aim of this study was to evaluate the community exercise program conducted by the WINDREF. Exercise improves overall health outcomes, cognition, and reduces mortality and morbidity $[3,4,8,13]$.Specifically, community-based exercise programs have shown efficacy in reducing NCDs in other nations, such as Untied States or Canada [10,11]. Grenada is experiencing an increase in NCDs also, likely due to obesity, smoking, alcohol, and inactivity.The obesity epidemic in Grenada is alarming. According to Word Health Organization, Grenada, Health Statistic Report, almost $22 \%$ of population is obese, with roughly $13.7 \%$ of men and $37 \%$ of women are of obese category. The exact statistics on NCDs for Grenada are not available in the literature; it can be safely assumed that obesity epidemic has led to having an increased risk of having NCDs in Grenada. Evaluating the efficacy of the community based exercise program,sponsored by Grenada Sports for Health program, is important and necessary to gauge its value in reducing the growing epidemic of obesity and possibly reducing a growing problem of NCDs in Grenada.

The evaluation was done measuring health outcomes of BMI and waist: hip ratio of participants in the sports for health program. BMI is often used as a surrogate measure for excess body fat, since 
it measures excess weight, however, studies have shown BMI is correlated to more direct measures of body fat[14,15]. Studies show an increased mortality risk associated with both a higher and lower range of BMI, making it an appropriate measure for assessing obesity and associated health risks[16]. While the interpretation of BMI is more useful for population characteristics and other more accurate methods to measure body fat (dual energy X-ray, bioelectrical impedance) exist[14], BMI is the most cost-effective and feasible in Grenada. A study also found measuring waist to hip ratio is a better indicator of cardiovascular risk than BMI[17], thus it was beneficial to assess waist to hip ratio among the participants.

The results of the study showed a significant change in both BMI and waist:hip ratio among participants over the period 2011 to 2014, which was then maintained by participants in 2016. The data suggests that, in the short term, Grenada Sports for health program, which is acommunity basedexercise program,is an effective tool for reducing BMI and waist: hip ratio. Other studies have also shown beneficial improvement in BMI with behavioural intervention over medications (sibutramine, orlistat)[18].There is also an association between household eating habits, soda consumption, and increased BMI[19-21].To address these concerns, dietary counselling, home based exercise regimens, and community basedexercise programs are utilized and studied.Home based exercise programs have shown a greater efficacy and adherence to regular exercise in the long run, however in the short term, community programs areconsidered better[22].Community based exercise programs serve as a venue for continuing improvements in participants once they feel they have met their goals. Such programs allow them to continue their efforts at their own pace and maintain their improved health measures. As our results show that participants maintained healthy BMI over time and showed greatest reduction in their BMI initially, attributing to community based exercise approach.

As many factors exist that result in increased BMI, obesity, and the development of chronic diseases, addressing them collectively would provide a greater benefit. Addressing healthy aspects of the individuals' lifestyle, nutrition, and implanting community basedexercise programs would further help alleviate the growing burden of non communicablechronic diseases in Grenada.Our study has limitations. There is no control group to compare the change in health indicators based on exercise approach; home versus community-based exercise program. Also, we had a roughly $40 \%$ attrition rate, as a result a loss to follow up. There is no data exist for individual matching between the two assessment dates. Although there was a loss to follow up and there is no matched data between the two groups. Another limitation of the study is that there is no data on NCDs among the participants, before and after the intervention.It is safely assumed, that participants with high BMI before the interventions were at higher risks of developing NCDs, such as CAD, OSA, and HTN[23].

\section{Conclusion}

This study aimed to evaluate the efficacy of the Grenada Sports for Health program, a community based exercise program, in improving health outcomes such as BMI and Waist to Hip ratios. The data obtained showed that the program was effective, especially from 2011 to 2014 with maintenance of physical measures in 2016. The implementation of Grenada Sports for Health Program and its community basedexercise approach can help improve BMI, obesity, and reducing the burden of non-communicable disease in Grenada.

\section{Acknowledgement}

Windward Islands Research and Education Foundation (WINDREF), Royal Grenada Police Force, Graduate Public Health Students of St. George's University.

\section{References}

1. Di Cesare M, Khang Y, Asaria P, Blakely T, Cowan M, et al. (2013) Inequalities in non-communicable diseases and effective responses. The Lancet 381(9866): 585-597.

2. Rasella D, Aquino R, Barreto ML (2013) Impact of income inequality on life expectancy in a highly unequal developing country: the case of Brazil. Journal of Epidemiology and Community Health 67(8): 661-666.

3. (2010) WHO Global status report on non-communicable diseases.

4. Lee IM, Shiroma EJ, Lobelo F, Puska P, Blair SN, et al. (2012) Effect of physical inactivity on major non-communicable diseases worldwide: an analysis of burden of disease and life expectancy. Lancet 380(9838): 219-229.

5. (2016) WHO. Physical activity.

6. Erickson K, Kramer A (2008) Aerobic exercise effects on cognitive and neural plasticity in older adults. British Journal Of Sports Medicine 43(1): 22-24.

7. Cassilahs R, R Viana V, Grassmann V, Santos R, Tufik S, et al. (2007) The Impact of Resistance Exercise on the Cognitive Function of the Elderly. Medicine \& Science In Sports \& Exercise 39(8): 1401-1407.

8. Middleton LE, Mitnitski A, Fallah N, Kirkland SA, Rockwood K (2008) Changes in cognition and mortality in relation to exercise in late life: a population based study. PloS One 3(9): e3124.

9. Okura T, Saghazadeh M, Soma Y, Tsunoda K (2013) Physical fitness, physical activity, exercise training and cognitive function in older adults. The Journal of Physical Fitness and Sports Medicine 2(3): 275-286.

10. Davis SK, Quarells R, Gibbons GH (2013) A comprehensive cardiovascular disease lifestyle treatment controlled trial among high-risk African Americans. Open Journal of Preventive Medicine 3(9): 526-533.

11. Desveaux L, Beauchamp M, Goldstein R, Brooks D (2014) Communitybased exercise programs as a strategy to optimize function in chronic disease: a systematic review. Medical Care 52(3): 216-226.

12. Ackermann RT, Cheadle A, Sandhu N, Madsen L, Wagner EH, et al. (2003) Community exercise program use and changes in healthcare costs for older adults. American Journal of Preventive Medicine 25(3): 232-237.

13. (2012) IOM (Institute of Medicine). An integrated framework for assessing the value of community-based prevention. The National Academies Press, Washington, USA.

14. (2014) Centers for Disease Control. Body Mass Index: Considerations for Practitioners.

15. Garrido-Chamorro RP, Sirvent-Belando JE, Gonzalez-Lorenzo M, MartinCarratala ML, Roche E (2009) Correlation between body mass index and body composition in elite athletes. Journal of Sports Medicine and Physical Fitness 49(3): 278-284.

16. Lewis CE, McTigue KM, Burke LE, Poirier P, Eckel RH, et al. (2009) Mortality, health outcomes, and body mass index in the overweight range. A science advisory from the American Heart Association. Circulation 119(25): 3263-3271.

17. Murray S (2006) Is waist-to-hip ratio a better marker of cardiovascular risk than body mass index? CMAJ : Canadian Medical Association Journal 174(3): 308. 
18. Whitlock E, O'Connor E, Williams S, Beil T, Lutz K (2010) Effectiveness of Primary Care Interventions for Weight Management in Children and Adolescents: An Updated, Targeted Systematic Review for the USPSTF. Pediatrics 125(2): 396-418.

19. Mâsse L, Blanck H, Valente M, Atienza A, Agurs-Collins T, et al. (2005) Association Between Self-Reported Household Practices and Body Mass Index of US Children and Adolescents, 2005. Preventing Chronic Disease p. 9.

20. Harrington S (2008) The role of sugar-sweetened beverage consumption in adolescent obesity: a review of the literature. J Sch Nurs 24(1): 3-12.

ISSN: 2574-1241

DOI: 10.26717/BJSTR.2018.06.001388

Satesh Bidaisee. Biomed J Sci \& Tech Res

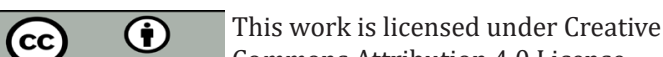

Submission Link: https://biomedres.us/submit-manuscript.php
21. Pereira M (2006) The possible role of sugar-sweetened beverages in obesity etiology: a review of the evidence. International Journal of Obesity 30: S28-S36.

22. Ashworth NL, Chad KE, Harrison EL, Reeder BA, Marshall SC (2005) Home versus center based physical activity programs in older adults. The Cochrane Database of Systematic Reviews 25(1): CD0040017.

23. (2014) WHO. Non-Communicable diseases.

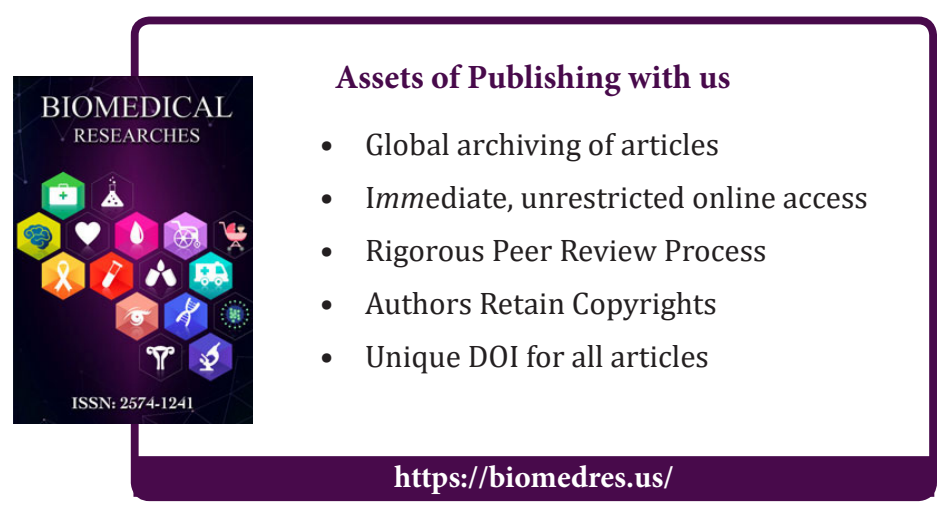

